Keywords: Shyness, Sociability, Social skills, Schizophrenia.

\title{
Stability of shyness, sociability, and social dysfunction in schizophrenia: A preliminary investigation of the influence of social skills training in a community-based stable outpatient sample
}

\author{
Michelle K. Jetha* \\ Louis A. Schmidt* \\ Joel O. Goldberg ${ }^{\star \star, * \star \star}$ \\ * Department of Psychology, Neuroscience \& \\ Behaviour, McMaster University \\ ** Hamilton Program for Schizophrenia \\ *** Department of Psychiatry and Behavioral \\ Neurosciences, McMaster University \\ CANADA
}

\begin{abstract}
Background: We previously noted a significantly higher degree of retrospective and contemporaneous shyness and sociability troubles in patients with schizophrenia than controls in a community-based stable outpatient sample and greater relative resting right frontal EEG activity (a trait marker of stress) in patients with schizophrenia who were shy.

Objective: Here we conducted a preliminary investigation to examine the stability of shyness and sociability in a sample of 37 stable outpatients with schizophrenia, some of whom were either exposed $(n=17)$ or not exposed $(n=20)$ to weekly social skills training over a seven month period.

Results: We found that shyness and sociability remained stable across all patients, although when the two groups were considered separately, only shyness remained stable in both groups. Sociability was not stable in those patients exposed to social skills training. We also found that, although measures of shyness indexed prior to exposure to the social skills training program significantly predicted pre- and post-social skills training measures, sociability was not related to pre- or post-social skills measures. Individuals with schizophrenia who scored high on trait shyness prior to exposure to social skills training still exhibited problems related to social adjustment following training.

Conclusions: The present study appears to be the first to examine the stability of trait-like shyness in schizophrenia. Findings are discussed in terms of their implications for understanding the stability of shyness and sociability and their role in the heterogeneity of symptom profiles and pervasive social dysfunction in community outpatients with schizophrenia.
\end{abstract}

Received 21 June 2006

Revised 26 December 2006

Accepted 16 January 2007 
One of the most disabling features and consequences of schizophrenia is a marked impairment of social skills ${ }^{1}$. Normal social interactions involve components of perception, assessment, and response to interpersonal cues ${ }^{2}$. Negative symptoms of schizophrenia (i.e., decreased spontaneous movements, paucity of expressive gestures and facial reactivity, flattened vocal intonation, poor eye contact, and social withdrawal) ${ }^{3}$ are especially destructive to normal social interaction and are inversely correlated with social skills performance ${ }^{4}$. These behavioral deficits are also associated with poor prognosis, cognitive impairments, and reduced functioning in social and occupational domains ${ }^{5}$.

Behavioral deficits in social functioning such as poor eye contact and social withdrawal are also characteristic of temperamental shyness $^{6,7}$, and parallel those behavioral deficits present in the symptom profile of schizophrenia. Although there is research suggesting that stable individual differences in personality do exist among people with schizophrenia $^{8}$ and can possibly influence the severity and symptomology of the pathology $9,10,11$, few studies have investigated shyness and related constructs as possible risk factors for social dysfunction in schizophrenia. One study by Flanagan ${ }^{12}$ measured shyness in hospitalized patients with schizophrenia and controls and reported that patients with schizophrenia experienced greater shyness than controls. More recently, we noted a higher degree of early and contemporaneous shyness and sociability troubles in patients than controls $^{13}$ and greater relative right resting frontal EEG activity (a trait marker of stress) in patients with schizophrenia who were shy ${ }^{14}$. These findings have important implications in light of research suggesting that early biological and behavioral antecedents of shyness and social withdrawal are identifiable in infants and young children, are linked to sensitivity of forebrain limbic and frontal cortical areas, and produce dysfunction in one's ability to regulate social stress ${ }^{6,7}$. It follows that both early and contemporaneous shyness may be implicated as risk factors for social dysfunction in schizophrenia.

A wealth of research exists concerning premorbid social functioning in schizophrenia that has been derived from both retrospective and longitudinal studies in an attempt to differentiate personality markers for developing psychosis. Poorer premorbid social adjustment in individuals with schizophrenia than controls has been evidenced in many of these investigations ${ }^{15-17}$. Premorbid adjustment was also related to social skill level within schizophrenia ${ }^{18}$. The New York High Risk Project (NYHRP), an ongoing longitudinal study profiling children at high risk for developing schizophrenia or schizophrenia spectrum related disorders, reported childhood attentional dysfunction and social isolation were more common in participants at risk for schizophrenia than those who did not go on to develop the disorder ${ }^{19}$. Follow-up investigations from the NYHRP also reported that individuals who developed schizophreniarelated psychosis in adulthood had presented with elevated negative symptomology and thought disorders in childhood ${ }^{20}$.

There is reason to believe that premorbid behavioral deficits including social withdrawal, attentional deficits, and social isolation are related to the disease process as is evidenced by correlations among negative symptoms and both functional and structural brain abnormalities in adult schizophrenia patients ${ }^{21}$. What appears critical and remains to be investigated is the influence of stable personality styles such as shyness and related constructs on symptom expression and social functioning in schizophrenia.

The purpose of the present study was to extend our previous findings that suggest shyness and sociability are identifiable in schizo- 
phrenia and are related to social functioning measures ${ }^{13}$ by exploring the stability of these trait-like constructs in this population. Here we conducted a preliminary investigation to examine: 1) the stability of shyness and sociability in a sample of 37 individuals with schizophrenia who were stable outpatients at a community-based treatment and rehabilitation program, 2) a convenience sample of a subset of 17 of the 37 patients who were exposed to a weekly social skills training program over a seven month period and compare them with the remaining 20 patients who were not exposed to social skills training on measures of pre- and post-shyness and pre- and post-sociability, and 3) pre- and post-shyness and pre- and post-sociability in relation to preand post-social skills measures only within the patients exposed to social skills training.

\section{Method}

\section{Participants}

The total study sample comprised 37 (27 males, 10 females) stable outpatients with a DSM-IV chart diagnosis of schizophrenia who ranged in age from 24 to 51 years $(\mathrm{M}=$ 41 years, $\mathrm{SD}=6.1$ years). All outpatients attended the Hamilton Program for Schizophrenia, a community-based outpatient treatment and rehabilitation program ${ }^{22}$. The psychiatric status of the patients was measured by the Positive and Negative Syndrome Scale (PANSS) ${ }^{3}$ and found to be comparable to stable community outpatients with schizophrenia. The PANSS was rated by a trained psychometrist and was conducted within a month of participation in the present study. All participants were stable outpatients, and a majority of them were on atypical neuroleptics and were diagnosed as paranoid or residual at the time of the study. Of the 37 outpatients, 17 were exposed to social skills training and 20 were not exposed to social skills training (see below). Relevant participant demographics for the two groups are presented in Table I. The two groups did not differ on any of the relevant demographic factors.

\section{Self-Report Measures}

Cheek and Buss Shyness and Sociability (CBSS) Scale. The CBSS $^{23,24}$ is a 10-item self-report questionnaire that indexes shyness

Table I

Demographic information for the social skills training and no social skills training groups in stable outpatients with schizophrenia

\begin{tabular}{|c|c|c|}
\hline Demographics & $\begin{array}{l}\text { Social Skills Training } \\
\qquad(n=17)\end{array}$ & $\begin{array}{l}\text { No Social Skills Training } \\
\qquad(n=20)\end{array}$ \\
\hline \multicolumn{3}{|l|}{ Sex } \\
\hline Male & 14 & 13 \\
\hline Female & 3 & 7 \\
\hline \multicolumn{3}{|l|}{ Mean (SD): } \\
\hline Age in years & $38.9 \quad(6.7)$ & $42.8 \quad(5.2)$ \\
\hline Education in years & $13.00(1.46)$ & $12.45(3.09)$ \\
\hline Age of onset & $21.13(5.66)$ & $19.70(3.23)$ \\
\hline Years since diagnosis & $17.94(8.96)$ & $23.05(5.93)$ \\
\hline \multicolumn{3}{|l|}{ PANSS } \\
\hline Positive & $13.0 \quad(4.7)$ & $11.7 \quad(4.8)$ \\
\hline Negative & $13.9 \quad(5.3)$ & $12.5 \quad(4.5)$ \\
\hline General & $28.5 \quad(7.2)$ & $25.8 \quad(6.2)$ \\
\hline Total & $55.1 \quad(14.3)$ & $49.9 \quad(13.6)$ \\
\hline
\end{tabular}


and sociability. The questionnaire contained the five highest-loaded shyness items ${ }^{25}$ from the original 13-item Cheek and Buss Shyness Scale, and the five sociability items from the original Cheek and Buss Sociability Scale ${ }^{23}$. The items refer to stable experiences or behaviors that occur during social interaction. Items from the shyness scale include: 'I find it hard to talk to strangers'; items from the sociability scale include: 'I like to be with people'. The items are answered on a zero to four metric, with the high scores reflecting shyness and sociability for the two scales. Reliability and validity for these two widelyused scales are reported elsewhere ${ }^{23,25,26}$.

The Social Relations Survey (SRS). The SRS is self-report inventory that comprises a 128-item true-false questionnaire to evaluate levels of social skills performance ${ }^{27}$. The measure provides scores on eight subscales representing constructs of interpersonal and empathic skills: Social Assertiveness--measuring the competence or skill of interacting with people and forming new relationships; Defense of Rights--rating the ability to defend one's rights and to refuse unjust or unwarranted demands involving violation of the individual's rights; Directiveness--pertaining to the ability to take charge, to direct the activities of others, and to exert influence on others; Positive Feeling Expression-measuring the ability to express approval or positive feeling toward another person; Confidence--rating the belief in one's own ability to succeed on tasks undertaken; Perceived Social Approval--pertaining to feeling accepted, liked, included, and approved of by significant others; Social Approval Need--pertaining to the motive to be popular, well-liked, respected, and held in high esteem; and Empathy--measuring the ability to take the viewpoint of the other person and to respond effectively to the perceived emotional experience of the other. These eight subscales then form two broad band factors: Social skills (comprising all six assertiveness scales: Social Assertiveness, Defense of Rights, Directiveness, Expression of Positive Feelings, Confidence, and Perceived Social Approval), and Empathy (comprising Social Approval Need and Empathy).

\section{Social Skills Training}

Intervention comprised structured weekly psycho-education delivered in a group-based format. There were 24 sessions conducted over the course of seven months, plus several 'day-trip' community outings for skills generalization. The content was derived mainly from the curriculum developed by Bellack and his colleagues ${ }^{28}$. The group was co-led by a predoctoral-level psychology intern, an occupational therapist, and an outpatient social skills training peer support worker who served as a role-play instructor.

\section{Procedures}

A convenience sample of 17 outpatients who were exposed to weekly social skills training were compared to the other 20 outpatients who were not exposed to the training. The CBSS was administered to all 37 patients pre- and post-exposure or no exposure to the seven-month social skills training. The SRS was administered only to the 17 patients who had undergone social skills training. (Note. Only patients who undergo social skills training at the Hamilton Program for Schizophrenia are administered the SRS. Accordingly, the analyses reported below involving the SRS data are on the 17 patients exposed to social skills training. We did not have SRS measures on the 20 patients not exposed to social skills training). 


\section{Results}

\section{Stability of Shyness and Socia- bility Across Patients}

We first examined the stability of shyness and sociability across all 37 patients. A Pearson correlation was computed separately for shyness and sociability across the seven month period. The correlations revealed that shyness $(\mathrm{n}=37, \mathrm{r}=.65, \mathrm{p}<.0005)$ and sociability $(\mathrm{n}=37, \mathrm{r}=.63, \mathrm{p}<.0005)$ remained highly stable across this period for all 37 outpatients.

\section{Influence of Social Skills Train- ing: Within-Patient Stability}

We next examined whether social skills training had any influence on pre- and postexposure shyness and sociability. Accordingly, we performed two sets of analyses. First, we examined mean differences between the patients exposed to social skills training to those not exposed separately on shyness and sociability scores collected pre- and postexposure. A series of between-subject $t$-tests failed to reveal any significant group differences on: pre-shyness measure (Social Skills Group $\mathrm{M}=11.2, \mathrm{SD}=3.9$ versus No Social Skills group $\mathrm{M}=8.7, \mathrm{SD}=4.5, \mathrm{t}(35)=1.88$, $\mathrm{p}>$ >05); post-shyness measure (Social Skills Group $\mathrm{M}=10.2, \mathrm{SD}=4.9$ versus No Social Skills group $\mathrm{M}=8.9, \mathrm{SD}=5.2, \mathrm{t}(35)=.77, \mathrm{p}$ $>$.05); pre-sociability measure (Social Skills Group $\mathrm{M}=11.5, \mathrm{SD}=3.6$ versus No Social Skills group $\mathrm{M}=12.3, \mathrm{SD}=4.8, \mathrm{t}(35)=.52$, $\mathrm{p}>.05$ ); and post-sociability measure (Social Skills Group $\mathrm{M}=12.7, \mathrm{SD}=2.5$ versus No Social Skills group $\mathrm{M}=12.4, \mathrm{SD}=5.4, \mathrm{t}(35)$ $=.23, \mathrm{p}>.05)$.

Second, we computed within-patient correlations to examine the relation between pre- and post-exposure shyness and sociability, separately within the two groups of patients. Figure 1 presents a scatterplot of these relations for each group.

As can be seen in Figure 1 (top panel), shyness remained stable from pre- to postfor those patients exposed to weekly social skills training $(\mathrm{n}=17, \mathrm{r}=.61, \mathrm{p}<.009)$ and those patients who were not exposed to social skills training $(\mathrm{n}=20, \mathrm{r}=.67, \mathrm{p}<$ .001 ) across the seven months. Whereas, sociability scores tended to change from pre- to post- for those patients exposed to weekly social skills training $(n=17, r=.17$, $\mathrm{p}>.05)$ and remained stable for those patients who were not exposed to social skills training $(\mathrm{n}=20, \mathrm{r}=.79, \mathrm{p}<.0005)$ across the seven months (see Figure 1, bottom panel).

\section{Within-patient Relations between Shyness, Sociability and Social Skills Measures}

We next computed a series of Pearson correlations to examine the within-patient relations between pre- and post-exposure shyness and sociability and pre- and postexposure social skills measures only for the patients exposed to the social skills training in relation to the two composite measures: Social Skills and Empathy.

Pre-exposure shyness was related to preexposure composite measure of Social Skills $(\mathrm{n}=17, \mathrm{r}=-.75, \mathrm{p}<.001)$, but not Empathy $(\mathrm{n}=17, \mathrm{r}=-.08, \mathrm{p}>.05)$. Increases in shyness were related to decreases in social skills, but not empathy, prior to exposure to social skills training. It is also important to note the pre-exposure shyness was related to all of the six individual subscales that comprised the Social Skills composite measure. These same patterns of relations 

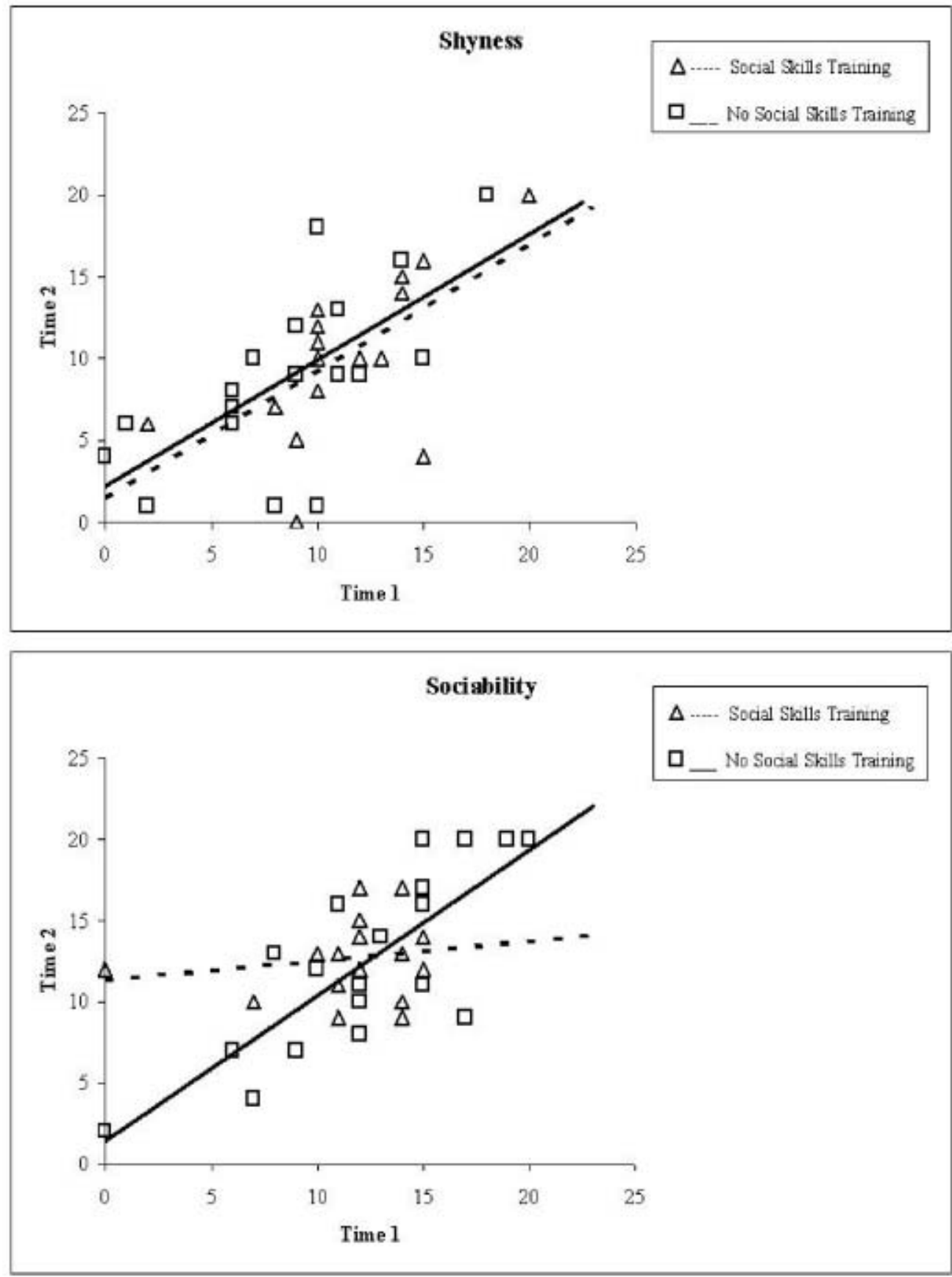

Figure 1. Scatterplots of the relation between pre- (Time 1) and post- (Time 2) shyness (top panel) and sociability (bottom panel) for stable outpatients with schizophrenia exposed to weekly social skills training over a seven month period $(n=17)$ and those who were not exposed to social skills training $(n=20)$ 
were also noted between pre-exposure shyness and post-exposure composite measure of social skills. Increases in shyness measured prior to social skills training were related to decreases in Social Skills measured post-exposure $(\mathrm{n}=17, \mathrm{r}=-.76$, $\mathrm{p}<.0005)$, but again not to Empathy $(\mathrm{n}=17$, $r=-.37, p>.05)$. Overall, these results suggest that shyness predicted levels of social skills pre- and post-exposure to social skills training and that exposure to social skills had little to no influence on the patient if he or she were shy. Individuals with schizophrenia who reported high degrees of shyness pre-exposure reported still having low social skills post-exposure.

It is also important to note that there were no significant relations noted between pre- and post-exposure sociability and preand post-exposure social skills composite measures, suggesting that sociability may play a different role in social skills in schizophrenia.

\section{Discussion}

We earlier noted a relation between shyness and social dysfunction in schizophrenia and suggested that shyness might be a possible risk factor for pervasive social dysfunction in individuals with this disorder ${ }^{13}$. Our further investigations have related shyness with greater relative right frontal EEG asymmetry (a correlate of anxious temperament ${ }^{14}$, suggesting that psychophysiological correlates of this trait-like construct may be identifiable in this population and may reflect vulnerabilities in stress regulation ${ }^{7}$. The purpose of this preliminary investigation was to extend our earlier findings by examining the stability of shyness and sociability across a seven month period and in response to a widely-used social skills intervention program ${ }^{28}$. Our findings suggest that not only is shyness identifiable in individuals with schizophrenia, but it appears to be robust and trait-like in nature as it remained stable in all patients regardless of exposure to social skills training. On the other hand, sociability appeared to remain stable only in patients who were not exposed to social skills training.

We also found that shyness before exposure was related to pre- and post-exposure social skills measures, suggesting that those individuals high on shyness showed deficits in social skills that were unchanged as a result of intervention. It is important to note that when considering the relation between shyness and the social relation subscales, analysis showed that shyness was only related to the six subscales that define social skill in terms of assertiveness and not to those measures that define empathy. Increased shyness in individuals with schizophrenia may predict social assertiveness, but not necessarily other important interpersonal skills involving empathy, perspective taking, and the need for social approval. These overall patterns of relations between personality and pre- and post-social skills measures were specific to the pre-exposure shyness measure and not the pre-sociability measure, suggesting that the sociability construct may be more malleable and less trait-like in nature than shyness in schizophrenia.

The present study is not without limitations. First, the results of this preliminary study are based on a relatively small sample size. The small sample size may limit generalizations to a broader population of individuals with schizophrenia. Future studies should attempt to replicate the present findings with a larger sample of adults with schizophrenia than used here. A second lim- 
itation concerns the reliance on self-report measures in general for patients with schizophrenia, which have some potential for distortion depending on the degree of psychotic symptoms. However, the relatively low levels of positive and negative symptom scores among our stable outpatient group as well as the stability of the self-report measures over time may minimize this concern. A third issue was that we failed to collect social skills measures in the patients who were not exposed to training. Accordingly, we did not have any direct measure as to whether the outpatients not exposed to training changed in their social skills during the seven months. The 17 patients exposed to social skills training were part of a convenience sample that allowed us to examine potential influences of social skills training on the stability of shyness and sociability. Although the purpose of the present preliminary study was not on the efficacy of social skills intervention per se, but rather on the stability of personality, future work may include social skills measures in the comparison group to examine the effectiveness of the training program for patients who differ on these personality variables. It is also important to note that failure to find changes in shyness and sociability following social skills training could be attributed to an ineffectiveness of this intervention strategy. However, this is unlikely, as this is a wellestablished and widely-used behavioral intervention. Another plausible explanation is that the intervention did not work on the measures we used because our measures may not have been sensitive enough to detect differences. Although sociability changed within the patient group, future work should replicate this finding and include other personality and social functioning measures to ensure that the influence of personality variables on social skills was specific to shyness.
While the heterogeneity of symptom profiles in individuals with schizophrenia has been previously examined in terms of other personality dimensions ${ }^{10,11}$, the present study appears to be the first to examine the stability of shyness in schizophrenia. Individuals with schizophrenia have been shown to possess stable personality profiles ${ }^{8}$, and certain maladaptive personality profiles may predate illness onset ${ }^{29,30}$. Here we replicated and extended these earlier findings in the extant literature by demonstrating that shyness, and to a lesser extent sociability, are identifiable and show stability across time and in response to some environmental influences in stable outpatients with schizophrenia. The present results also indicate that the identification and tracking of shyness and sociability may have important implications for choices of clinical strategies that seek to modify social skills in individuals with this disorder and who might benefit most from such interventions. Such rehabilitation programs are known to have a positive influence not only on the patients but on the emotional well-being of the caregivers as well ${ }^{31}$.

The results of the present study implicate early and contemporaneous shyness as possible risk factors for social dysfunction. Stable shyness in healthy individuals is known to predict social adjustment problems ${ }^{7}$. Shyness appears to be a stable personality feature in schizophrenia, perhaps less open to change than other features of personality, and is associated with trait-like resting frontal EEG activation ${ }^{14}$. Thus, future research should consider how temperamental shyness "colors" various aspects of socio-emotional processing in patients with schizophrenia given deficits in these processes are profound in individuals with schizophrenia and are known to play an important role in social functioning. 


\section{Acknowledgements}

This research was funded in part by operating grants from the Social Sciences and Humanities Research Council (SSHRC) of Canada awarded to LAS and the CSVR Foundation awarded to JOG, and a Pre-doctoral Scholarship from the Natural Sciences and Engineering Research Council (NSERC) of Canada awarded to MKJ under the direction of LAS. Portions of this paper were presented at the $155^{\text {th }}$ Annual Meeting of the American Psychiatric Association, Philadelphia, PA, May, 2002 and the 2005 International Congress on Schizophrenia Research, Savannah, GA, April, 2005. We wish to thank Susan Gensey for her contributions as clinical rater and Dr. Peter Cook (Executive Director), and the staff and clients at the Hamilton Program for Schizophrenia.

\section{References}

1. Morrison RL, Bellack AS. Social functioning of schizophrenic patients: Clinical and research issues. Schizophr Bull 1987; 13: 715-725.

2. Wallace CJ. The social skills training project of the mental health clinical research center for the study of schizophrenia. In: Curran JP, Monti PM, editors. Social skills training: A practical handbook for assessment and treatment. New York: Guilford Press; 1982. p. 57-89.

3. Kay SR, Fiszbein A, Opler LA. The Positive and Negative Syndrome Scale (PANSS) Rating Manual. New York: Albert Einstein College of Medicine; 1987.

4. Jackson HJ, Minas IH, Burgess PM, Joshua SD, Charisiou J, Campbell IM. Is social skills performance a correlate of schizophrenia subtypes? Schizophr Res 1989; 2: 301-309.

5. Davidson L, McGlashan TH. The varied outcomes of schizophrenia. Can J Psychiatry 1997; 42: 34-43.

6. Schmidt LA, Fox NA. Individual differences in childhood shyness: Origins, malleability, and developmental course. In: Cervone D, Mischel W, editors. Advances in
Personality Science. New York: Guilford Press; 2002. p. 83-105.

7. Schmidt LA, Polak CP, Spooner AL. Biological and environmental contributions to childhood shyness: A diathesis-stress model. In: Crozier WR, Alden LE, editors. The Essential Handbook of Social Anxiety for Clinicians. United Kingdom: John Wiley \& Sons; 2005. p. 33-55.

8. Kentros M, Smith TE, Hull J, McKee M, Terkelsen K, \& Capalbo C. Stability of personality traits in schizophrenia and schizoaffective disorder: A pilot project. J Nerv Ment Dis 1997; 185: 549-555.

9. Davidson L, Straus JS. Sense of self in recovery from severe mental illness. Br J Med Psychol 1992; 65: 131145.

10. Guillem F, Bicu M, Semkovska M, Debruille JB. The dimensional symptom structure of schizophrenia and its association with temperament and character. Schizophr Res 2002; 56: 137-147.

11. Lysaker PH, Bell MD, Kaplan E, Greig TC, Bryson GJ. Personality and psychopathology in schizophrenia: The association between personality traits and symptoms. Psychiatry 1999; 62: 36-48.

12. Flanagan R. Shyness, egocentricity, and psychopathology: Their relationships among nonhospitalized individuals and mental hospital patients. Psychol Rep 1992; 70: 995-1004.

13. Goldberg JO, Schmidt LA. Shyness, sociability and social dysfunction in schizophrenia. Schizophr Res 2001; 48: 347-352.

14. Jetha MK, Schmidt LA, Goldberg JO. Resting frontal brain activation asymmetry and shyness and sociability in schizophrenia: A pilot study of a communitybased stable outpatient sample. Int J Neurosci 2007 (In press).

15. Cannon M, Jones P, Gilvarry C, Rifkin L, McKenzie K, Foerster A, Murray RM. Premorbid social functioning in schizophrenia and bipolar disorder: Similarities and differences. Am J Psychiatry 1997; 154: 1544-1550.

16. Lewine RRJ, Watt NF, Fryer JH. A study of childhood social competence, adult premorbid competence, and psychiatric outcome in three schizophrenic subtypes. J Abnorm Psychol 1978; 87: 294-302.

17. Rodriguez-Solano JJ, Gonzalez de Chavez M. Premorbid adjustment and previous personality in schizophrenic patients. Eur J Psychiat 2005; 19: 243-254.

18. Mueser KT, Bellack, AS, Morrison RL, Wixted JT. Social competence in schizophrenia: Premorbid adjustment, social skill, and domains of functioning. J Psychiatr Res 1990; 24: 51-63. 
19. Erlenmeyer-Kimling L, Cornblatt BA, Rock D, Roberts S, Bell M, West A. The New York High-Risk Project: Anhedonia, attentional deviance, and psychopathology. Schizophr Bull 1993; 19: 141-153.

20. Ott SL, Allen J, Erlenmeyer-Kimling L. The New York High-Risk Project: Observations on the rating of early manifestations of schizophrenia. Am J Med Genet 2001; 105: 25-27.

21. Gruzelier JH. Functional neuropsychophysiological asymmetry in schizophrenia: A review and reorientation. Schizophr Bull 1999; 25: 91-120.

22. Whelton C, Pawlick J, Cook PE. Growing with clients in a psychosocial rehabilitation program. Psychiatr Rehab 1999; 22: 290-293.

23. Cheek JM, Buss AH. Shyness and sociability. J Pers Soc Psychol 1981; 41: 330-339.

24. Cheek JM. The revised Cheek and Buss Shyness Scale. Unpublished manuscript, Wellesley College, Wellesley, MA. 1983.

25. Bruch MA, Gorsky JM, Collins TM, Berger PA. Shyness and sociability reexamined: A multi-component analysis. J Pers Soc Psychol 1989; 57: 904-915.

26. Hopko DR, Stowell J, Jones WH, Armento ME, Cheek JM. Psychometric properties of the Revised Cheek and Buss Shyness Scale. J Pers Assess 2005; 84: 185-192.

27. Lorr M, Youniss RP, Stefic CE. An inventory of social skills. J Pers Assess 1991; 57: 506-520.
28. Bellack AS, Mueser KT, Gingerick S, Agresta J. Social skills training for schizophrenia: A step-by-step approach. New York: Guilford Press; 1997.

29. Cuesta MJ, Peralta V, Caro F. Premorbid personality in psychoses. Schizophr Bull 1999; 25: 801-811.

30. Done DJ, Crow TJ, Johnstone EC, Sacker A. Childhood antecedents of schizophrenia and affective illness: Social adjustment at ages 7 and 11. BMJ 1994; 309: 699703.

31. Koukia E, Madianos MG. The effect of rehabilitation of schizophrenic patients on their family atmosphere and the emotional well-being of caregivers. Eur J Psychiatry $2005 ; 19: 55-64$.

Address for correspondence:

Dr. Louis A. Schmidt

Department of Psychology, Neuroscience \& Behaviour, McMaster University, Hamilton, Ontario

Canada L8S 4K1

phone: 905-525-9140, ext. 23028; fax: 905-529-6225

email: schmidtl@mcmaster.ca

Dr. Joel Goldberg

Hamilton Program for Schizophrenia

102-350 King Street East

Hamilton, Ontario

Canada L8N 3 Y3

phone: 905-525-2832; fax: 905-546-0055

email: jgoldber@mcmaster.ca 